\title{
Attention deficit hyperactivity symptoms and disorder (ADHD) among African children: a review of epidemiology and co-morbidities
}

\section{MO Bakare}

Child and Adolescent Unit, Federal Neuro-Psychiatric Hospital, New Haven, Enugu, Enugu State, Nigeria

\begin{abstract}
Objective: To review literature from Africa on the epidemiology of ADHD as well attention deficit hyperactivity symptoms and associated co-morbid conditions among African children. Method: A literature search was done through Pubmed/MEDLINE and Google Scholar using then following terms, "attention deficit", "hyperactivity disorders", "epidemiology", "co-morbid conditions", "Africa". Nine studies met the inclusion criteria with four studies coming from South Africa, two each from Democratic Republic of Congo and Nigeria respectively and one from Ethiopia. Results: The prevalence of ADHD varied with rates of between 5.4\% and $8.7 \%$, amongst school children, 1.5\% amongst children from the general population between 45.5\% to 100.0\% amongst special populations of children with possible organic brain pathology. Common associated co-morbid conditions were oppositional defiant disorder, conduct disorder as well as anxiety/depressive symptoms. Conclusion: Published data from Africa is limited. For effective healthcare policy further studies are needed to define the magnitude and burden of ADHD and other childhood neurodevelopmental disorders in Africa.
\end{abstract}

Keywords: Attention deficit; Hyperactivity; Symptoms; Disorders; Africa

Received: 20/06/201 1

Accepted: 25/09/201 1

doi: http://dx.doi.org/10.4314/ajpsy.v15i5.45

\section{Introduction}

A debate exists as to whether attention deficit hyperactivity disorder (ADHD) might be a cultural construct. ${ }^{1-5}$ The opinion that geographical location may have some influence on epidemiology of ADHD and Attention Deficit Hyperactivity Symptoms remains ${ }^{1,3}$, despite a few studies having concluded, with some level of caution however, that culture and geographical location may have little or no influence on the epidemiology of ADHD world-wide. 6,7

In their systematic review of literature on the world-wide prevalence of $\mathrm{ADHD}$, Polanczyk et $\mathrm{al}^{6}$ found a prevalence of about five percent. However, they cautioned against generalization of their findings, especially in geographical

\section{Correspondence}

Dr MO Bakare

Child and Adolescent Unit, Federal Neuro-Psychiatric Hospital,

New Haven, Enugu, Enugu State, Nigeria

email: mobakare2000@yahoo.com regions of Africa and Middle East because these regions contributed very few studies to their meta-analysis. ${ }^{6}$

Though controversy persists about the influence of culture and geographical locations on the epidemiology of ADHD, there has been general agreement amongst studies that method of assessment, diagnostic criteria employed, source of information and inclusion of impairments in functioning in operational definition criteria greatly influence the prevalence rates documented in the literature. . $^{1-7}$

While childhood ADHD has been extensively researched in Europe and North America, very few studies are available in the African sub-region. Since the publication on world-wide prevalence of ADHD in $2007^{6}$, a number of studies had emerged from Africa on the epidemiology of $\mathrm{ADHD}$, Attention Deficit Hyperactivity symptoms and associated comorbidities among African children. ${ }^{7-11}$ Physical and psychological conditions can occur co-morbidly with ADHD and some of these co-morbidities have been documented in studies from Africa. ${ }^{8-11}$ 
There has been no prior review of the prevalence of ADHD and ADHD symptoms documented in different countries of sub-Saharan Africa. The current content therefore constitutes a review of past epidemiological studies on ADHD, Attention Deficit Hyperactivity symptoms and associated co-morbid conditions documented subSaharan Africa.

\section{Method}

The review comprised a literature search of the following databases - Pubmed/MEDLINE and Google Scholar, using the following terms; "attention deficit", "hyperactivity disorders", "epidemiology", "co-morbid conditions", "Africa". Literature relevant to epidemiology of ADHD, ADHD symptoms and associated co-morbidities coming from Africa were included. References included in the selected literatures which were relevant to Africa in area of epidemiology of ADHD and ADHD symptoms were also reviewed. Some of the authors of these studies were also contacted to clarify some issues that were not clear in their studies. All studies addressing epidemiology of ADHD, ADHD symptoms and co-morbidities in Africa and carried out among children who are below 18 years were included in the review. The last updated search was undertaken in September 2011.

Ethical clearance for this study was obtained from the Institutional Review Board of Federal Neuropsychiatric Hospital, Enugu, Enugu State, Nigeria.

\section{Results}

The search yielded nine (9) studies relevant to aspects of epidemiology and co-morbidity of ADHD and ADHD symptoms in Africa. ${ }^{7-15}$ While four of the studies were from South Africa $9,10,12,13$, two studies each were from Nigeria ${ }^{7,11}$ and Democratic Republic of Congo ${ }^{8,14}$ respectively, and only one study from Ethiopia. ${ }^{15}$ Four studies addressed epidemiology of ADHD among population of school children in Africa ${ }^{7,12,13,14}$, one study among population of children in the general community. ${ }^{15}$ Three studies ${ }^{9,10,11}$ addressed epidemiology of ADHD symptoms among special populations of children with HIV infection, tuberculosis meningitis, and intellectual disability in Africa, while one study dealt solely with co-morbid conditions. ${ }^{8}$ The details of the reviewed literature are shown in Table I.

\section{Discussion}

Epidemiology of Attention Deficit Hyperactivity Disorders (ADHD) among populations of school children in Africa The prevalence of ADHD among school children according to studies conducted in Africa ranges between 5.4\% and 8.7\%, 7,12,13,14 The studies coming from South Africa documented a prevalence of about five percent, which concurred with the finding of a prevalence of about five percent in the meta-analysis study of word-wide prevalence of ADHD by Polanczyk et al. ${ }^{6}$ The only epidemiological study among school children coming from the Democratic Republic of Congo documented a prevalence of 6.0\%, while the only epidemiological study coming from Nigeria among school children revealed a prevalence of $8.7 \%$.

There were multiple sources of information for making the diagnosis of ADHD in these studies with all of the studies making use of information from both the parents and the teachers albeit employing different diagnostic instruments. $7,12,13,14$

\section{Epidemiology of ADHD among children in the general population sample}

The rate of primary school enrolment in African countries had been noted to range from about thirty to ninety five percent, with much lower rates in sub-Saharan African countries. ${ }^{16}$ Therefore the prevalence studies of ADHD carried out among sub-Saharan African children in primary schools ${ }^{7,12,13,14}$ might not be representative of the actual prevalence in the general population. Unfortunately, only one study had looked at prevalence of ADHD among children selected from a general population sample in Africa. ${ }^{15}$ Ashenafi et $\mathrm{ll}^{15}$ found the prevalence of ADHD among children from the general population in a district in

\section{Table I: Country of Origin and Population of Children Studied in the Reviewed Literature}

\begin{tabular}{|l|l|l|}
\hline \multicolumn{2}{|l|}{ Table : Country of Origin and Population of Children Studied in the Reviewed Literature } \\
\hline Author/s & Countries of Origin & Population of Children Studied \\
\hline Meyer (1998) & South Africa & School Children \\
\hline Meyer et al (2004) & South Africa & School Children \\
\hline Kashala et al (2005) & Democratic Republic of Congo & School Children \\
\hline Kashala et al (2006) & Democratic Republic of Congo & School Children \\
\hline Adewuya \& Famuyiwa (2007) & Nigeria & Children from the General Population \\
\hline Ashenafi et al (2001) & Ethiopia & Children with Tuberculosis Meningitis \\
\hline Wait et al (2002) & South Africa & Children with HIV Infection \\
\hline Zeegers et al (2010) & South Africa & Children with Intellectual disability \\
\hline Bakare et al (2010) & Nigeria & \\
\hline
\end{tabular}


Ethiopia to be $1.5 \%$. The prevalence of $1.5 \%$ in this study ${ }^{15}$ is by far lower than the range of $5.4 \%$ to $8.7 \%$ documented in the studies conducted among primary school children in South Africa, Nigeria and Democratic Republic of Congo. $7,12,13,14$

The reasons for the apparent disparity in prevalence between the community and school studies are not quite clear. There is however a possibility that symptoms of ADHD, especially in-attentive sub-type are made more noticeable and apparent by the school environment compared to the home or community environment where high levels of concentration might not be required in normal routine tasks. Another possibility is the difference in diagnostic instruments employed in assessment before arriving at a diagnosis of ADHD. However, this possibility is more remote because the diagnostic instruments employed in all the studies $7,12,13,14,15$ factored in diagnostic criteria specified in the World Health Organization (WHO) International Classification of Diseases, Tenth Edition (ICD10) ${ }^{17}$ and Diagnostic and Statistical Manual of Mental Disorders, Fourth Edition (DSM-IV). ${ }^{18}$

\section{Epidemiology of Attention Deficit Hyperactivity Symptoms in special population of children with possible organic brain pathology in Africa}

Three studies addressed epidemiology of Attention Deficit Hyperactivity Symptoms in special population of children.911 Zeegers et $\mathrm{al}^{9}$ assessed the epidemiology of ADHD among HIV infected South African children. They found a combined prevalence of ADHD to be $88 \%$ in the studied population, with inattentive type accounting for $26 \%$, hyperactive type, 38\% and combined type, $24 \% .{ }^{9}$ In another study, Wait et $\mathrm{al}^{10}$ explored the association between tuberculosis meningitis and Attention Deficit Hyperactivity Symptoms. They found that one hundred percent (100\%) of children that had just recovered from tuberculosis meningitis had Attention Deficit Hyperactivity Symptoms. Based on this finding, they speculated that ADHD may be a long term complication of tuberculosis meningitis. ${ }^{10}$ Both studies ${ }^{9,10}$ further reinforce the possibility of organic brain conditions as aetiological factors in ADHD among African children. Bakare et $\mathrm{al}^{11}$ looked at problem behaviour among children with intellectual disability. The study found a prevalence of $45.5 \%$ hyperactivity symptoms among children with intellectual disability. ${ }^{11}$

One of the proposed aetiological factors of $\mathrm{ADHD}$ is brain damage resulting from circulatory, toxic, metabolic, mechanical or physical insults. ${ }^{19}$ The high prevalence of Attention Deficit Hyperactivity Symptoms ranging from about forty five to one hundred percents as documented by the aforementioned studies ${ }^{9,10,11}$ supports the notion that possible brain damage is an important aetiological explanation for ADHD among African children.9,10,11 The prevalence distribution of ADHD/ ADHD Symptoms in different population of children in Africa is shown in Table II.

\section{Epidemiology of $A D H D$, gender differences and co- morbidities in Africa}

Regarding gender, all the studies on epidemiology of ADHD among African children reviewed agreed on a higher prevalence of ADHD or Hyperactivity symptoms among boys compared to girls. ${ }^{7,9,10,11,12,13,14,15}$

The most commonly reported co-morbid conditions associated with ADHD among African children were Oppositional Defiant Disorder (ODD) and Conduct Disorder. ${ }^{7,8,9,14,15}$ Anxiety and depressive symptoms may also co-exist with ADHD among African Children. ${ }^{7}$ How frequently these co-morbid conditions co-exist with ADHD varied according to the population sample of children studied.

\section{Limitations}

It is important to emphasize that the existing literature in the field of ADHD from Africa is relatively sparse which limits the ability to realistically generalize the findings of this review as the prevalent trend of $\mathrm{ADHD}$ and attention deficit hyperactivity symptoms among African children. A further limitation may be that the search approach did not identify all relevant studies. Notwithstanding these limitations, it is imperative to examine the existing data to determine the knowledge gaps related to the epidemiology of $\mathrm{ADHD}$ and attention deficit hyperactivity symptoms among African children. It is believed that the findings of this review will guide future epidemiological studies of $\mathrm{ADHD}$ and subsequent policy making in Africa.

\section{Conclusion}

The prevalence of ADHD in Africa based on studies coming from the continent ranges between 5.4\% and 8.7\% among populations of school children ${ }^{7,12,13,14}$ with the prevalence being much lower among a population of children from the general community where a prevalence of $1.5 \%$ was documented. ${ }^{15}$

In addition, studies from Africa affirmed that Attention Deficit Hyperactivity symptoms do complicate various conditions, characterized by possible organic brain pathology, in children.8,9,10,20 The need to prevent these conditions, which have the potential for damaging effects on the developing brain, among African children is thus imperative.

\begin{tabular}{|l|l|}
\hline \multicolumn{2}{|l|}{ Table II: Prevalence of ADHD/ ADHD Symptoms amongst different populations of African children } \\
\hline Population of Children & Prevalence of ADHD/ADHD Symptoms \\
\hline School Children & $5.4 \%-8.7 \%$ \\
\hline Children from General Population & $1.5 \%$ \\
\hline Children from Special Population (With Possible Organic Brain Pathology) & $45.5 \%-100.0 \%$ \\
\hline
\end{tabular}


Limited research studies are available from the continent addressing the epidemiology of ADHD and other childhood neuro-developmental disorders. Such studies are essential for formulating policy on intervention and healthcare service delivery. This is a further reflection of less attention paid to issues of childhood neuro-developmental disorders by Governments of the countries in Africa.

If adequate health service planning is to be put in place on the African continent, further studies are required to potentially more accurately reflect the magnitude and burden of ADHD and other childhood neuro-developmental disorders in Africa.

\section{Acknowledgements}

The author thanked Dr. Polanczyk for providing list of African literatures reviewed in their study. The author is also grateful to Dr. Adewuya for his assistance in providing a reprint of their study.

\section{References}

1. Anderson JC. Is childhood hyperactivity the product of Western culture? Lancet. 1996; 348: 73 - 74 .

2. Bird HR. The diagnostic classification, epidemiology and crosscultural validity of ADHD, In Attention Deficit Hyperactivity Disorder: State of the Science; Best Practices. Edited by Jensen, PCJ, Kingston, N.J, Civic Research Institute, 2002.

3. Faraone SV, Sergeant J, Gillberg C, Biederman J. The worldwide prevalence of ADHD: is it an American condition? World Psychiatry 2003; 2: $104-113$

4. Timimi S, Taylor E. ADHD is best understood as a cultural construct. Br J Psychiatry 2004; 184: 8 - 9 .

5. Rappley MD. Attention deficit-hyperactivity disorder. N Engl J Med 2005; 352: 165 - 173 .

6. Polanczyk G, de Lima MS, Horta BL, Biederman J, Rhode LA. The worldwide prevalence of ADHD: A systematic review and metaregression analysis. Am J Psychiatry 2007; 6; 164: 942 - 948.

7. Adewuya AO, Famuyiwa OO. Attention deficit hyperactivity disorder among Nigerian primary school children: Prevalence and co-morbid conditions. Eur Child Adolesc Psychiatry 2007; 16: $10-15$

8. Kashala E, Lundervold A, Sommerfelt K, Tylleskar T, Elgen I. Coexisting symptoms and risk factors among African school children with hyperactivity-inattention symptoms in Kinshasa,
Congo. Eur Child Adolesc Psychiatry 2006: 15(5): 292 - 299

9. Zeegers I, Rabie H, Swanevelder S, Edson C, Cotton M, vanToorn R. Attention deficit hyperactivity and oppositional defiance disorder in HIV-infected South African children. J Trop Pediatr 2010; $56(2): 97-102$.

10. Wait JW, Stanton L, Schoeman JF. Tuberculosis meningitis and attention deficit hyperactivity disorder in children. J Trop Pediatr 2002; 48(5): $294-299$.

11. Bakare MO, Ubochi VN, Ebigbo PO, Orovwigho AO. Problem and pro-social behavior among Nigerian children with intellectual disability: the implication for developing policy for school based mental health programs. Italian Journal of Pediatrics 2010; 36: 37

12. Meyer A. Attention Deficit/Hyperactivity Disorder among North Sotho speaking primary school children in South Africa: Prevalence and sex ratios. Journal of Psychology in Africa 1998; 8: $186-195$

13. Meyer A, Eilertsen DE, Sundet JM, Tshifularo JG, Sagvolden T. Cross cultural similarities in ADHD-like behavior amongst South African primary school children. South African Journal of Psychology 2004; 34: 123 - 139 .

14. Kashala E, Tylleskar T, Elgen I, Kayembe KT, Sommerfelt K. Attention deficit and hyperactivity disorder among school children in Kinshasa, Democratic Republic of Congo. Afr Health Sci 2005; 5(3): $172-181$

15. Ashenafi $Y$, Kebede D, Desta M, Alem A. Prevalence of mental and behavioural disorders in Ethiopian children. East Afr Med J 2001; 78(6): $308-311$.

16. ChildInfo/Unicef. Primary school enrolment and attendance in Africa (2003 to 2008)

http://wWw.childinfo.org/education_primary.php

17. World Health Organization (WHO). The International Classification of Diseases, Tenth Edition (ICD - 10). WHO, Geneva, Switzerland; 1992.

18. American Psychiatric Association (APA). Diagnostic and Statistical Manual of Mental Disorders, Fourth Edition (DSM - IV). American Psychiatric Publishing, Washington DC; 1994.

19. Sadock BJ, Sadock VA. Kaplan \& Sadock's Concise Textbook of Child and Adolescent Psychiatry. Wolters Kluwer/Lippincott Williams \& Wilkins, 2009; Chapter 7: 80

20. Idro R, Kakooza-Mwesige A, Balyejjussa S, Mirembe G, Mugasha C, Tugumisirize J, et al. Severe neurological sequelae and behaviour problems after cerebral malaria in Ugandan children. BMC Res Notes 2010; 3: 104

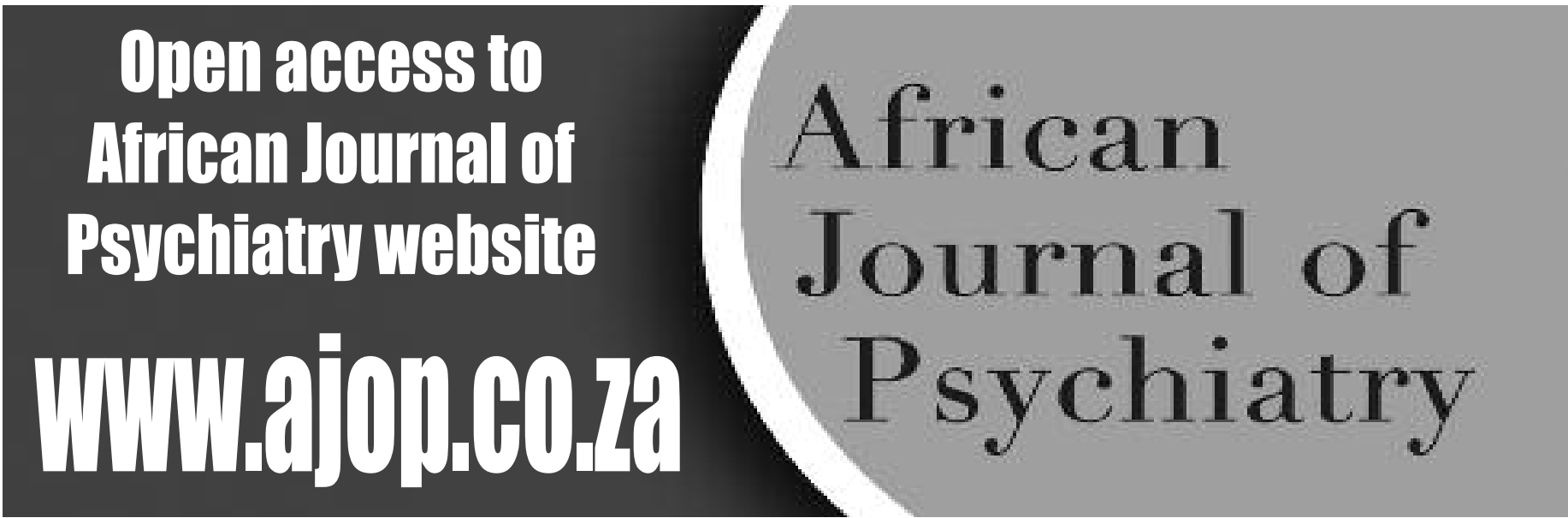

